\title{
Sussan Babaie, Talinn Grigor (eds.). Persian Kingship and Architecture, Strategies of Power in Iran from the Achaemenids to the Pahlavis
}

\section{Rémy Boucharlat}

\section{(2) OpenEdition}

Journals

Édition électronique

URL : http://journals.openedition.org/abstractairanica/45843

DOI : $10.4000 /$ abstractairanica. 45843

ISBN : 1961-960X

ISSN : 1961-960X

Éditeur :

CNRS (UMR 7528 Mondes iraniens et indiens), Éditions de l'IFRI

\section{Référence électronique}

Rémy Boucharlat, « Sussan Babaie, Talinn Grigor (eds.). Persian Kingship and Architecture, Strategies of Power in Iran from the Achaemenids to the Pahlavis », Abstracta Iranica [En ligne], Volume 37-38-39 | 2018, document 4, mis en ligne le 30 décembre 2018, consulté le 28 septembre 2020. URL : http:// journals.openedition.org/abstractairanica/45843; DOI : https://doi.org/10.4000/abstractairanica. 45843

Ce document a été généré automatiquement le 28 septembre 2020.

Tous droits réservés 


\title{
Sussan Babaie, Talinn Grigor (eds.). Persian Kingship and Architecture, Strategies of Power in Iran from the Achaemenids to the Pahlavis
}

\author{
Rémy Boucharlat
}

\section{RÉFÉRENCE}

Sussan Babaie, Talinn Grigor (eds.). Persian Kingship and Architecture, Strategies of Power in Iran from the Achaemenids to the Pahlavis. London-New York: IB Tauris, 2016, XXIV-260 p., nombreuses ill. et $32 \mathrm{pl}$. coul.

Selon l'introduction des éditrices, le concept de la royauté en Iran, transcendant l'ethnicité, la religion et la politique, renvoie avant tout à Eranshahr, le pouvoir ou l'autorité royal sur le territoire des Aryens (Iraniens) depuis les Achéménides jusqu'à l'époque moderne. Margaret Cool Root, «Achaemenid Imperial Architecture : Performative Porticoes of Persepolis» (p. 1-64) décrit longuement la terrasse de Persepolis et les monuments que porte celle-ci, comme un lieu de cérémonie avant tout. Elle insiste sur la grande salle dite Apadana et ses portiques, marquant spécialement celui de l'ouest ouvrant sur la plaine, laquelle était peu bâtie selon l'A. «agrarian lanscape ». Cette partie est l'éminente représentation du pouvoir, que l'on retrouvera plus tard à Isfahan (cf. cr de O. Ramble). Matthew P. Canepa, «Dynastic Sanctuaries and the Transformation of Iranian Kingship between Alexander and Islam » (p. 64-117) fonde sa démonstration sur les continuités dans le temps, près d'un millénaire, et dans l'espace iranien, de l'Anatolie à l'Indus, une approche qu'il a développée largement ailleurs ces dernières années. (cf. cr L. Colliva). L'article du regretté Lionel Bier, "The Sasanian Palaces and Their Influence in Early Islam » (p. 119-133) est repris de Ars Orientalis 23, 1993. Il est de ce fait un peu daté pour l'interprétation des complexes palatiaux et/ou religieux de Bishapur et de l'Imarat-e 
Khosrow dans l'ouest de l'Iran, car plusieurs études, parfois contradictoires, ont paru depuis sur ces monuments. Son approche de la continuité de l'époque sassanide à l'époque omeyyade reste toutefois opérante.

Dans "The Footsteps of the Sasanians: Funerary Architecture and Bavandid Legitimacy» (p. 135-173), Melanie Michailidis, disparue avant la publication du livre, montre que le Tabaristan, région isolée par les montagnes, a constitué un conservatoire de la culture iranienne de l'époque sassanide, à travers l'exemple de trois tours funéraires du XIe et début XIIe siècle. Sussan Babaie, «Sacred Sites of Kingship: The Maydan and Mapping the Spatial-Spiritual Vision of the Empire in Safavid Iran » (p. 174-217) montre la nouvelle théorie politique des Safavides qui, tenant compte des exigences du chi'sme, organisent différemment les villes dans lesquelles la place publique, maydan, joue un rôle de premier plan. Pour Talinn Grigor, «Kingship Hybridized, Kingship Homogeneized: Revivalism under the Qajar and the Pahlavi Dynasties» (p. 218-254), que ce soit sous les Qajars, malgré les revers militaires puis le déclin politique, ou sous les Pahlavis dans le courant de la modernisation de l'Iran, l'art et l'architecture font un retour à la grandeur passée, principalement par référence aux réalisations de l'empire achéménide.

La continuité ou le retour volontaire aux formes du passé, surtout achéménide et sassanide, prennent toutefois en compte les apports des différentes époques intermédiaires, par exemple les éléments islamiques à l'époque safavide.

\section{AUTEURS}

\section{RÉMY BOUCHARLAT}

UMR 5133 CNRS-Université de Lyon 